\title{
Infinite-Dimensional Symplectic Capacities and a Squeezing Theorem for Hamiltonian PDE's
}

\author{
Sergej B. Kuksin \\ IPPI, Institute for Information Transmission Problems, Moscow, Russia
}

Received: 21 October 1993/in revised form: 6 April 1994

\begin{abstract}
We study partial differential equations of hamiltonian form and treat them as infinite-dimensional hamiltonian systems in a functional phase-space of $x$-dependent functions. In this phase space we construct an invariant symplectic capacity and prove a version of Gromov's (non)squeezing theorem. We give an interpretation of the theorem in terms of the "energy transition to high frequencies" problem.
\end{abstract}

\section{Introduction}

Lebesgue measure as an invariant of symplectic transformations for a long time has been used as a useful tool to study hamiltonian systems (we mention the Poincaré recurrence theorem as an example of its elegant application). Recently M. Gromov [G] discovered another invariant which he called "Darboux width" and which was lately developed by I. Ekeland-H. Hofer as "symplectic capacity." The new invariant has already found several highly nontrivial applications, starting from Gromov's sequeezing theorem ${ }^{1}$ (the "Darboux width" was created as a tool to prove this result). Some alternative definitions for the I. Ekeland-H. Hofer capacity ("mostly," but not "absolutely" equivalent to the initial one) were given later by C. Viterbo and H. Hofer-E. Zehnder. Below we use the definition from [HZ1, HZ2], where references to the previous works also can be found.

On the other hand, it was realized during the last two decades, mostly starting with the works of V.E. Zakharov (see e.g. [Za]) that partial differential equations without dissipation of energy can be written as hamiltonian systems in an infinite-dimensional phase-space $Z$ of $x$-dependent functions. Thus a natural question arises if infinite-dimensional symplectic invariants can be constructed and used to study hamiltonian PDE's.

It is well-known that the Lebesgue measure $d x$ has no infinite-dimensional analogy. But the Gibbs measure $e^{-H(x)} d x$, where $H$ is the hamiltonian of the

${ }^{1}$ Stating that "a symplectic camel cannot go through the eye of a needle" (V.I. Arnold, [1]). 
equation we study, could have it. ${ }^{2}$ Such a measure was first constructed by L. Friedlander $[\mathrm{F}]$ for the $\varphi^{4}$-equation

$$
\ddot{u}=u_{x x}-u^{3}, \quad u=u(t, x), \quad x \in \mathbb{T}^{1} ;
$$

lately invariant Gibbs measures were obtained for some other $x$-one-dimensional equations with coercive hamiltonians by P. Zhidkov, H. McKean-K. Vaninski and J. Bourgain (see [Bo] and references therein).

In this paper we construct for hamiltonian PDE's the second invariant symplectic capacity. For each open subset $\mathcal{O}$ of the phase-space $Z$ of $x$-dependent vector-functions we define its capacity $c(\mathcal{O})$ in such a way that the following properties are fulfilled:

1) translational invariance:

$$
c(\mathcal{O})=c(\mathcal{O}+\xi) \text { for } \xi \in Z
$$

2) monotonocity:

$$
c\left(\mathcal{O}_{1}\right) \geqq c\left(\mathcal{O}_{2}\right) \text { if } \quad \mathscr{O}_{1} \supset \mathcal{O}_{2}
$$

3) 2-homogeneity:

$$
c(\tau \mathcal{O})=\tau^{2} c(\mathcal{O})
$$

4) nontriviality:

$$
0<c(\mathcal{O})<\infty \quad \text { if } \mathcal{O} \neq \emptyset \text { is bounded. }
$$

Our definition of the capacity $c(\mathcal{O})$ is based on finite-dimensional approximations of 0 . Thus the capacity $c$ inherits the very important normalization of the finite-dimensional symplectic capacity:

$$
c\left(B_{r}\right)=c\left(\Pi_{r}^{(k)}\right)=\pi r^{2},
$$

where $B$ is an $r$-ball in $Z$ and $\Pi_{r}^{(k)}$ is the cylinder based on an $r$-disc in the plane spanned by the vectors $\varphi_{k}^{+}, \varphi_{k}^{-}$from a fixed Darboux basis $\varphi_{1}^{ \pm}, \varphi_{2}^{ \pm}, \ldots$ in $Z$. (Possibly the ball and the cylinder are not centered at zero.)

We prove that the capacity $c$ is preserved by the flow-maps $S_{t}$ of a (nonautonomous) hamiltonian equation, provided that

$$
S_{t}=\text { linear operator + compact smooth operator, }
$$

where the linear operator is the direct sum of rotations in the planes spanned by $\varphi_{j}^{+}$and $\varphi_{j}^{-}(j=1,2, \ldots)$ and compactness of the nonlinear term "agrees with the basis $\left\{\varphi_{j}^{ \pm}\right\}$" (these two assumptions hold trivially if the linear operator is the identity map).

The assumption (1) does not hold for general hamiltonian PDE, but still it holds for some important equations. For example, for

Nonlinear string equation

$$
\ddot{u}=u_{x x}+p(u ; t, x), \quad u=u(t, x), x \in \mathbb{T}^{1},
$$

\footnotetext{
2 The Gibbs measure depends on the hamiltonian. Therefore it can be used to study autonomous equations only.
} 
where $p$ is a smooth function which has at most a polynomial growth as $|u| \rightarrow \infty$;

Quadratic nonlinear wave equation

$$
\ddot{u}=\Delta u+a(t, x) u+b(t, x) u^{2}, \quad x \in \mathbb{T}^{2} ;
$$

Nonlinear membrane equation

$$
\ddot{u}=-\Delta^{2} u+p(u ; t, x), \quad x \in \mathbb{T}^{2} ;
$$

Schrödinger equation

$$
-i \dot{u}=-\Delta u+V(x) u+\left[\frac{\partial}{\partial \bar{U}} G(U, \bar{U} ; t, x)\right] * \xi, \quad U=u^{*} \xi, \quad x \in \mathbb{T}^{n},
$$

where $u * \xi$ is the convolution with a fixed real function $\xi$ and $G$ is a real-valued smooth function.

Usually there is an arbitrariness in choosing a phase-space for a hamiltonian PDE. In contrast, to construct the invariant capacity one should take for a phasespace of the equation the Hilbert space distinguished by the property that some Hilbert basis of the space is in the same time a Darboux basis for the corresponding symplectic structure. For a nonlinear PDE such a distinguished phase-space $Z$ is essentially unique. In particular, for Eq. (2) the phase-space is $Z=H^{1 / 2}\left(\mathbb{T}^{1}\right) \times H^{1 / 2}\left(\mathbb{T}^{1}\right)$, formed by the vector-functions

$$
\left(u(x),\left(-\hat{o}^{2} / \partial x^{2}+1\right)^{-1 / 2} \dot{u}(x)\right) .
$$

For (3) and (4) one should take $Z=H^{1 / 2}\left(\mathbb{T}^{2}\right) \times H^{1 / 2}\left(\mathbb{T}^{2}\right)$ and $Z=H^{1}\left(\mathbb{T}^{2}\right) \times H^{1}\left(\mathbb{T}^{2}\right)$; for (5) the space should be $Z=L_{2}\left(\mathbb{T}^{n} ; \mathbb{C}\right)$.

The flow-maps $S_{t}$ of Eq. (2)-(5) preserve the capacities of sets till the blow-up time. In particular, due to properties 2) and 5) of the capacity $c$ we get

Squeezing Theorem. If

$$
S_{t}\left(B_{R}\right) \subset \Pi_{r}^{(k)}
$$

for some ball $B_{R}$ and cylinder $\Pi_{r}^{(k)}$, then $r \geqq R$.

For Eq. (5) we have $\varphi_{s}^{+}=\varphi_{s}(x), \varphi_{s}^{-}=i \varphi_{s}(x)$, where $\left\{\varphi_{s}\right\}$ is a complete system of real eigenfunctions of $-\Delta+V(x)$. So

$$
u(t, x)=\sum u_{s}^{+}(t) \varphi_{s}^{+}+u_{s}^{-}(t) \varphi_{s}^{-}=\sum\left(u_{s}^{+}+i u_{s}^{-}\right)(t) \varphi_{s},
$$

and $\left\{p_{s}=u_{s}^{+}+i u_{s}^{-}\right\}$are the complex Fourier coefficients of the solution $u(t, x)$. The theorem implies that if $\left|p_{k}(t)\right|<r$ for all solutions with $u(0, \cdot) \in B_{R}$, then $r \geqq R$.

Another immediate consequence of the theorem is that a bounded solution of the equation cannot be "uniformly asymptotically stable" as $t \rightarrow \infty$, because for any $\rho$-neighbourhood $B_{\rho}$ of the initial point diameter of the set $S_{t}\left(B_{\rho}\right)$ cannot tend to zero.

The theorem is also connected with the following important question: does the energy of nonlinear conservative oscillations spread to higher frequencies? A possible mathematical reformulation of this physical question is the following.

Provide the phase-space of $x$-dependent vector-functions with some Hilbert norm $\|\cdot\| \sim$. Denote the corresponding Hilbert space as $\tilde{Z}$ and its Hilbert basis as 
$\left\{\tilde{\varphi}_{j}^{ \pm}\right\}$. Take a ball $B_{R}=\{\|z\| \sim<R\}$ in the space of initial data and consider the problem: is it true that for fixed $k$ "part of energy leaves $k$-th mode":

$$
S_{T}\left(B_{R}\right) \subset\left\{\sum p_{j} \tilde{\varphi}_{j}^{+}+q_{j} \tilde{\varphi}_{j}^{-} \mid p_{k}^{2}+q_{k}^{2}<\rho^{2}\right\}=\Pi_{\rho}
$$

for some $T>0, \rho<R$ ?

If $\tilde{Z}=Z$, then (7) is the squeezing, which is impossible due to the theorem.

To explain why we treat (7) as the spread of energy, suppose that the phasespace of the system we consider is the space of pairs of $x$-dependent functions with $x \in \mathbb{T}^{n}=\mathbb{R}^{n} /(2 \pi \mathbb{Z})^{n}$. Fix $d \geqq 0$ and choose the tilde-basis equal to $\left\{\tilde{\varphi}_{s}^{ \pm}(x) \mid s \in \mathbb{Z}^{n}\right\}$, where

$$
\tilde{\varphi}_{s}^{+}(x)=\langle s\rangle^{-d}\left(\varphi_{s}(x), 0\right), \quad \tilde{\varphi}_{s}^{-}(x)=\langle s\rangle^{-d}\left(0, \varphi_{s}(x)\right),
$$

$\langle s\rangle=1+|s|$ and $\left\{\varphi_{s}(x)\right\}$ is the usual trigonometric basis of $L_{2}\left(\mathbb{T}^{n}\right)$. For a vector-function denote by $z_{s}^{ \pm}$the coefficients of its decomposition in the basis $\left\{\left(\varphi_{s}(x), 0\right),\left(0, \varphi_{s}(x)\right)\right\}$ (i.e., its Fourier-coefficients) and denote by $E_{s}=z_{s}^{+^{2}}+z_{s}^{-2}$ the energy of the $s^{\text {th }}$ mode. Below we draw idealised pictures of the energy distribution for the points from $B_{R}$ (at left) and from $\Pi_{\rho}$ with $k=1$ (at right):

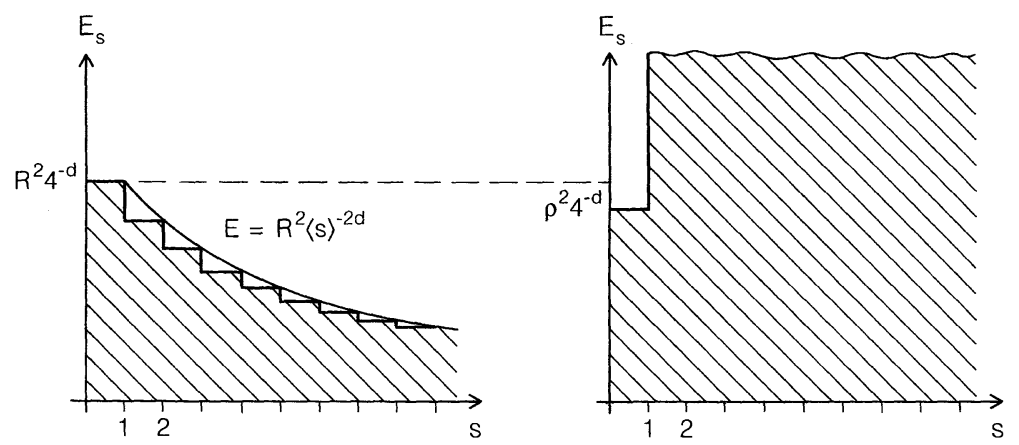

So if $\rho<R$ and the map $S_{T}$ sent the left set inside the right one, then a part of the energy indeed left the first mode. For more information on the energy-transition subject we refer the reader, say, to $[\mathrm{P}]$.

For Eqs. (2)-(5) above the distinguished phase-spaces $Z$ have the bases of the form (8) with $d=\frac{1}{2}, \frac{1}{2}, 1,0$ correspondingly. The question if the "squeezing" (7) is possible can be also stated with the distinguished phase-spaces $Z$ replaced by some smoother spaces. The most interesting seems the case of energetic spaces. For Eqs. (2)-(5) these are respectively Sobolev spaces $H^{1}, H^{1}, H^{2}, H^{1}$ (having the bases of the form (8) with $d=1,1,2,1)$. In such a case we do not know if (7) is possible or not for general Eqs. (2)-(5). But for Eq. (2) with a time-dependent analytic function $p(u)$ of the form

$$
p(u)=-m u+a u^{2}+b u^{3}+O\left(u^{4}\right),
$$

where $m>0$, the answer again is "no" if $\rho$ in (7) is sufficiently small. The reason is that for Eq. (2), (9) has in rich supply small-amplitude time-quasiperiodic solutions. See $[\mathrm{BK}]$ for the case $a=0, b=\mathrm{const} \neq 0$ and $[\mathrm{K}]$ for the case $m=m(x)$, where 
$m(\cdot)$ is a "typical function," even in $x \cdot{ }^{3}$ In particular, (2) has time-periodic solutions which lie in $B_{R}$ near the plane $\mathbb{R} \varphi_{k}^{+} \oplus \mathbb{R} \varphi_{k}^{-} .^{4}$

\section{Capacities of Finite-Dimensional Sets}

We provide the linear space $\mathbb{R}^{2 n}=\mathbb{R}_{p}^{n} \times \mathbb{R}_{q}^{n}$ with the usual symplectic structure $d p \wedge d q$; for a smooth function $f(p, q)$ we denote by $V_{f}$ the corresponding hamiltonian vectorfield.

Given $m>0$ and an open domain $\mathcal{O} \subset \mathbb{R}^{2 n}$ we call a function $f \in C^{\infty}(\mathcal{O})$ $m$-admissible if

i) $0 \leqq f \leqq m$;

ii) $f$ vanishes in a nonempty subdomain of $\mathcal{O}$;

iii) $f \equiv m$ in a neighborhood of $\partial \mathcal{O}$.

Following $[\mathrm{HZ} 1, \mathrm{HZ} 2]$ we define the capacity $c_{2 n}(\mathcal{O})$ of the domain $\mathcal{O}$ as

$c_{2 n}(\mathcal{O})=\inf \left\{m_{*} \mid\right.$ for each $m>m_{*}$ and each $m$-admissible $f$ in $\mathcal{O}$ the vectorfield $V_{f}$ has a nontrivial periodic solution of period $\left.\leqq 1\right\}$.

Clearly $c_{2 n}$ is a monotonic symplectic invariant:

$$
c_{2 n}\left(\mathcal{O}_{1}\right) \leqq c_{2 n}\left(\mathcal{O}_{2}\right) \quad \text { if } \quad \mathcal{O}_{1} \subset \mathcal{O}_{2}
$$

and

$$
c_{2 n}(\mathcal{O})=c_{2 n}(\phi(\mathcal{O}))
$$

if $\phi: \mathcal{O} \rightarrow \mathbb{R}^{2 n}$ is a symplectomorphism.

Denote by $B_{r}$ the ball

$$
B_{r}=\left\{\left.(p, q)|| p\right|^{2}+|q|^{2}<r^{2}\right\},
$$

and by $\Pi_{r}$ the cylinder

$$
\Pi_{\boldsymbol{r}}=\left\{(p, q) \mid p_{1}^{2}+q_{1}^{2}<r^{2}\right\} .
$$

The following equalities gives the main property of the capacity $c_{2 n}$ :

$$
c_{2 n}\left(B_{r}\right)=c_{2 n}\left(\Pi_{r}\right)=\pi r^{2}
$$

(see $[\mathrm{HZ} 1, \mathrm{HZ} 2]$ for a proof).

An immediate consequence of (1.1)-(1.3) is the famous squeezing theorem of $\mathbf{M}$. Gromov [G]: the ball $B_{r}$ can be symplectically embedded into the cylinder $\Pi_{R}$ only if $R \geqq r$.

Take any $n$-vector $r=\left(r_{1}, \ldots, r_{n}\right), 0<r_{j} \leqq \infty$, where some $r_{j}$ is finite. Then

$$
r:=\min _{1 \leqq j \leqq n} r_{j}=r_{j_{o}}<\infty
$$

${ }^{3}$ More exactly, [BK, K] provide (2), (9) with time-quasiperiodic, $x$-periodic solutions which are even or odd in $x$ since theorems of these works are applicable to even-periodic and odd-periodic boundary conditions (which are equivalent to Dirichlet and Neumann boundary conditions on the half-period).

${ }^{4}$ These solutions form symplectic ribs which prevent the symplectic camel from the first footnote to go through the eye of a needle (cf. Sect. 1 in [A]). 
with some $1 \leqq j_{0} \leqq n$. The ellipsoid

$$
B(\boldsymbol{r})=\left\{(p, q) \mid \sum r_{j}^{-2}\left(p_{j}^{2}+q_{j}^{2}\right)<1\right\}
$$

contains the ball $B_{r}$ and is contained in the cylinder $\left\{p_{j_{0}}^{2}+q_{j_{0}}^{2}<r^{2}\right\}$. So we get from (1.3) (and (1.1), (1.2)) that

$$
c_{2 n}(B(r))=\pi r^{2} .
$$

\section{Symplectic Hilbert Spaces}

Let $Z$ be a Hilbert space with the scalar product $\langle\cdot, \cdot\rangle$ and a Hilbert basis $\left\{\varphi_{j}^{ \pm} \mid j \geqq 1\right\}$. For $n \in \mathbb{N}$ we denote by $Z^{n}$ the linear envelope of the vectors $\left\{\varphi_{j}^{ \pm} \mid 1 \leqq j \leqq n\right\}$ and denote by $\Pi^{n}$ the natural projector

$$
\Pi^{n}: Z \rightarrow Z^{n} .
$$

We also denote $Z_{n}=Z \ominus Z^{n}$, use the decomposition of $Z$,

$$
Z=Z^{n} \oplus Z_{n},
$$

and write accordingly $z \in Z$ as

$$
z=\left(z^{n}, z_{n}\right), \quad z^{n} \in Z^{n}, \quad z_{n} \in Z_{n} .
$$

We define the skewsymmetric linear operator $J$,

$$
J: Z \rightarrow Z, \quad \varphi_{j}^{ \pm} \mapsto \mp \varphi_{j}^{\mp},
$$

and supply $Z$ with a symplectic structure by means of the 2-form

$$
\omega=\langle J d z, d z\rangle .^{5}
$$

We take a selfadjoint (possibly unbounded) linear operator $A$ such that

$$
A \varphi_{j}^{ \pm}=\lambda_{j} \varphi_{j}^{ \pm} \quad \forall j
$$

(the operator is selfadjoint in $Z$ with the natural domain of definition) and consider a hamiltonian

$$
f=\frac{1}{2}\langle A z, z\rangle+h(z ; t),
$$

where $h$ is a smooth in $Z \times \mathbb{R}$ function. The corresponding hamiltonian equation has the form

$$
\dot{z}=J(A z+\nabla h(z ; t)) .
$$

By (2.2) the hamiltonian operator $J A$ is diagonal in the complex basis $\left\{\frac{1}{\sqrt{2}}\left(\varphi_{j}^{+} \pm i \varphi_{j}^{-}\right)\right\}$with the eigenvalues $\left\{ \pm i \lambda_{j}\right\}$, and defines a group of isometries $\left\{e^{t J A} \mid t \in \mathbb{R}\right\}$ of the space $Z$,

$$
\left\|e^{t J A}\right\|_{Z, Z}=1 \quad \forall t
$$

${ }^{5}$ that is, $\omega[\xi, \eta]=\langle J \xi, \eta\rangle$. 
Remark. The assumption (2.2) concerning Eq. (2.4) is not very restrictive, see Part 6 for concrete examples. Besides, this assumption can be achieved if the "unbounded linear part" $J A_{u}$ of Eq. (2.4) has a discrete spectrum which is semisimple and imaginary - we can put the operator $J A_{u}$ to the normal form (2.2) with some Darboux basis $\left\{\varphi_{j}^{ \pm}\right\}$and introduce a Hilbert structure in $Z$ with $\left\{\varphi_{j}^{ \pm}\right\}$as a Hilbert basis. The "bounded linear part" $J A_{b}$ can be added to the nonlinear term $J \nabla h$, so it may contain Jordan cells and hyperbolic eigenvalues.

We say that a continuous curve $z(t) \in C\left(T_{1}, T_{2} ; Z\right)$ is a solution of (2.4) in $Z$ if for $T_{1} \leqq t \leqq T_{2}$,

$$
z(t)=e^{\left(t-T_{1}\right) J A} z(0)+\int_{T_{1}}^{t} e^{(t-\tau) J A} J \nabla h(z(\tau) ; \tau) d \tau .
$$

The integral in the r.h.s. is well-defined due to (2.5).

We denote by $S_{T_{1}, T_{2}}$ the operator which sends $z\left(T_{1}\right)$ to $z\left(T_{2}\right)$ (possibly $\left.T_{2}<T_{1}\right)$ and abbreviate $S_{0, t}=S_{t}$.

Remark. Define $Z_{A}$ as a Hilbert space with the norm $\|z\|_{A}^{2}=\left\langle\left(\mathbf{1}+A^{2}\right)^{-1} z, z\right\rangle_{Z}$. Then $Z \subset Z_{A}$ and the linear map

$$
A: Z \rightarrow Z_{A}
$$

is bounded. In particular, the solution (2.6) is a $C^{1}$-curve in $Z_{A}$ and defines a strong solution of Eq. (2.4) in this space (both sides of (2.4) coincide as continuous curves in $Z_{A}$ ).

For a Hilbert space $Z_{-}$we write

$$
Z<Z_{-}
$$

if $Z$ is compactly embedded in $Z_{-}$and $\left\{\varphi_{j}^{ \pm}\right\}$is an orthogonal basis of $Z_{-}$(not an orthonormal one!). Clearly, the space $Z$ is dense in $Z_{-}$.

We identify the adjoint space $Z^{*}$ with $Z$. Then $\left(Z_{-}\right)^{*}$ can be identified with a subspace $Z_{+}$of $Z, Z_{+}<Z$. So we have

$$
Z_{+}<Z<Z_{-} \text {. }
$$

If $\|\cdot\|_{-}$is the Hilbert norm of $Z_{-}$, then

$$
\left\|e^{t J A}\right\|_{-,-}=1,
$$

and similarly with $Z_{+}$.

By $B_{R}(Z)$, or $B_{R}$ if the space $Z$ is fixed, we denote the ball $\{z \mid\|z\|<R\}$. We also denote

$$
h_{t}(z)=h(z ; t)
$$

and impose the following compactness assumption: for some triad $Z_{+}<Z<Z_{-}$as above the function $h$ can be extended to a $C^{2}$-smooth function on $Z_{-} \times \mathbb{R}$. Then the gradient map $\nabla h_{t}: Z \rightarrow Z$ can be extended to maps

$$
\nabla h \in C^{1}\left(\mathbb{R} \times Z_{-}, Z_{+}\right), \quad \nabla h \in C^{\infty}(\mathbb{R} \times Z, Z) .
$$

In particular, the map

$$
\mathbb{R} \times Z \rightarrow Z_{+}, \quad(t, z) \mapsto \nabla h_{t}(z)
$$


is bounded (sends bounded sets to bounded) because the embedding $Z \rightarrow Z_{\text {- }}$ is compact. We suppose also that the map

$$
\mathbb{R} \times Z_{-} \rightarrow Z, \quad(t, z) \mapsto \nabla h_{t}(z) \quad \text { is bounded . }
$$

Definition 1. For an open domain $\mathcal{O} \subset Z$ and $T>0$ we write

$$
\tau(\mathcal{O}) \geqq T
$$

if solutions $u(t)$ of (1.4) exist for $0 \leqq t \leqq T$ for all $u(0)$ from some neighbourhood of $\overline{\mathcal{O}}$ in $Z$ (bar means closure).

Lemma 1. Suppose that the assumptions (2.2) and (2.8)-(2.8") hold. Let $\mathcal{O} \subset Z$ be a domain such that $\tau(\mathcal{O}) \geqq T>0$ and for each $R>0$ and $u(0) \in \mathcal{O} \cap B_{R}$ the solution $u(t), 0 \leqq t \leqq T$, does not leave some larger ball $B_{R^{\prime}}, R^{\prime}=R^{\prime}(R)$. Then the flow-maps

$$
S_{t}: u(0) \mapsto u(t), \quad 0 \leqq t \leqq T,
$$

are smooth diffeomorphisms of the form

$$
S_{t}=e^{t J A}\left(I+\widetilde{S_{t}}\right)
$$

where

$$
\tilde{S}_{t}(z)=\int_{0}^{t} e^{-\tau J A} J \nabla h\left(S_{\tau} z, \tau\right) d \tau
$$

Proof. It is sufficient to prove the statement for $\mathcal{O}$ replaced by $\mathcal{O} \cap B_{R}$ with arbitrary $R>0$. In Appendix 1 below we prove (in a traditional way) that the maps $S_{t_{1}, t_{2}}(u)$ for $u \in \mathcal{O} \cap B_{R}, 0 \leqq t_{1}, t_{2} \leqq T$, exist if $\left|t_{1}-t_{2}\right| \leqq \tau(R, T)$ and are diffeomorphisms. We can replace $\tau(R, T)$ by $\tau^{\prime}=\tau\left(R^{\prime}, T\right) \leqq \tau(R, T)$ and write $S_{t}$ as

$$
S_{t}=S_{t_{n-1}, t_{n}} \circ S_{t_{n-2}, t_{n-1}} \circ \cdots \circ S_{t_{0}, t_{1}},
$$

where $t_{n}=t, t_{0}=0$ and $\left|t_{j}-t_{j-1}\right| \leqq \tau^{\prime}$. The representation (2.9) readily follows from the integral equation (2.6) which holds for $z(t)=S_{t}(z)$.

The differential $d S_{T}\left(z_{0}\right)$ of the map $S_{T}$ sends a vector $v_{0}$ to $v(T)$, where $v(t)$ solves the linearised equation

$$
\dot{v}=J\left(A v+d \nabla h_{t}(z(t)) v\right)
$$

with $v(0)=v_{0}$. The curve $\{z(t) \mid 0 \leqq t \leqq T\}$ is compact in $Z$. Thus for all $0 \leqq t \leqq T$

$$
\left\|d \nabla h_{t}(z(t))\right\|_{Z, Z} \leqq C(T, z(\cdot)),
$$

and we have

$$
\|v(t)\| \leqq C_{1}(T, z(\cdot))\|v(0)\|, \quad 0 \leqq t \leqq T .
$$

Lemma 2. Under the assumption of Lemma 1 the flow maps $S_{t}$ are symplectic:

$$
S_{t}^{*} \omega=\omega \text {. }
$$

Proof. We should check that for any two vectors $v_{10}, v_{20}$ and $0 \leqq t \leqq T$,

$$
S_{t}^{*} \omega\left[v_{10}, v_{20}\right]=\text { const . }
$$

That is, we should check that

$$
l(t):=\left\langle J v_{1}(t), v_{2}(t)\right\rangle=\mathrm{const},
$$

where $v_{j}(t)$ is the solution of $(2.10)$ with $v_{j}(0)=v_{j 0}, j=1,2$. 
Denote

$$
l^{N}(t)=\left\langle J v_{1}(t), \Pi^{N} v_{2}(t)\right\rangle
$$

We have

$$
\begin{aligned}
\frac{d}{d t} l^{N}(t)= & \left\langle J \dot{v}_{1}, \Pi^{N} v_{2}\right\rangle+\left\langle J v_{1}, \Pi^{N} \dot{v}_{2}\right\rangle \\
= & \left\langle J^{2}\left(A v_{1}+d \nabla h_{t}(z) v_{1}\right), \Pi^{N} v_{2}\right\rangle \\
& +\left\langle J v_{1}, \Pi^{N} J\left(A v_{2}+d \nabla h_{t}(z) v_{2}\right)\right\rangle \\
= & -\left\langle d \nabla h_{t}(z) v_{1}, \Pi^{N} v_{2}\right\rangle+\left\langle v_{1}, \Pi^{N} d \nabla h_{t}(z) v_{2}\right\rangle .
\end{aligned}
$$

Thus,

$$
\begin{aligned}
l(t)-l(0) & =\lim _{N \rightarrow \infty}\left(l^{N}(t)-l^{N}(0)\right) \\
& =\lim _{N \rightarrow \infty} \int_{0}^{t}\left(\left\langle d \nabla h_{\tau}(z) v_{2}, \Pi^{N} v_{1}\right\rangle-\left\langle d \nabla h_{\tau}(z) v_{1}, \Pi^{N} v_{2}\right\rangle\right) d \tau=0,
\end{aligned}
$$

due to Lebesgue theorem, because by (2.11) the function under the integral is bounded by some $(t, N)$-independent constant and pointwise tends to zero.

We shall study Eq. (2.4) for $u(0)=u_{0} \in \mathcal{O} \subset Z$ and $0 \leqq t \leqq T$, where $\tau(\mathcal{O}) \geqq T$. In addition we suppose that the maps $S_{t}: \mathcal{O} \rightarrow Z$ are uniformly bounded for $0 \leqq t \leqq T$. That is, for each $R>0$ there exists $R^{\prime}$ such that

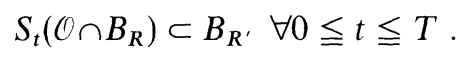

To study the solutions with $u_{0} \in \mathcal{O} \cap B_{R}$ we can replace $h_{t}(u)$ by

$$
\tilde{h}_{t}(u)=g\left(\|u\|_{-}^{2}\right) h_{t}(u),
$$

where the function $g \in C_{0}^{\infty}(\mathbb{R})$ is such that $g(r)=1$ for $|r| \leqq R^{\prime 2}$. Denote by $j: Z_{-} \rightarrow Z_{+}$the duality isomorphism:

$$
\langle u, v\rangle_{-}=\langle j u, v\rangle \text {. }
$$

Then

$$
\nabla g\left(\|u\|_{-}^{2}\right)=2 g^{\prime}\left(\|u\|_{-}^{2}\right) j u,
$$

and the function $\tilde{h}$ meets $(2.8)-\left(2.8^{\prime \prime}\right)$. The equation with the transformed hamiltonian $\tilde{h}$ has the same solutions for $u(0) \in \mathcal{O} \cap B_{R}$ and $0 \leqq t \leqq T$.

We say that the hamiltonian $f$ is admissible if it satisfies (2.2), (2.8)-(2.8") for some triad $Z_{+}<Z<Z_{-}$; it is admissible for $0 \leqq t \leqq T$ and $u_{0} \in \mathcal{O} \subset Z$ if also $T \leqq \tau(\mathcal{O})$ and (2.12) holds for each $R$.

Definition 2. A symplectomorphism $\Phi: Z \supset \mathcal{O} \rightarrow Z$ is called elementary if it is the $S_{T}$-map for some hamiltonian equation which is admissible for $0 \leqq t \leqq T$ and $u_{0} \in \mathcal{O}$.

Each elementary symplectomorphism $\Phi=S_{t}$ admits the representation (2.9), where the map $\widetilde{S_{t}}$ is compact by the assumption (2.8). Each compact map can be approximated by finite-dimensional maps. Below we need a symplectic version of this statement:

Lemma 3. For each elementary $\Phi$, each $\varepsilon>0$ and $R<\infty$ there exists $N$ such that

$$
\Phi(u)=e^{T J A}\left(I+\tilde{\Phi}_{\varepsilon}\right)\left(I+\tilde{\Phi}_{N}\right)(u)
$$


for $u \in \mathcal{O} \cap B_{R}(Z)$, where $\left(I+\widetilde{\Phi}_{\varepsilon}\right)$ and $\left(I+\widetilde{\Phi}_{N}\right)$ are smooth symplectomorphisms of $Z$ such that

$$
\left\|\tilde{\Phi}_{\varepsilon}(u)\right\| \leqq \varepsilon \quad \text { for } \quad u \in\left(I+\tilde{\Phi}_{N}\right)\left(\mathcal{O} \cap B_{R}\right),
$$

and in terms of the decomposition (2.1)

$$
\widetilde{\Phi}_{N}\left(z^{N}, z_{N}\right)=\left(\tilde{\Phi}_{N}^{0}\left(z^{N}\right), z_{N}\right) .
$$

For a proof see Appendix 2.

\section{Symplectic Capacities for Hilbert Space}

For an open nonempty domain $\mathcal{O} \subset Z$ and $n \geqq 1$ we denote

$$
\mathcal{O}^{n}=\mathcal{O} \cap Z^{n} \text {, }
$$

and observe that

$$
\partial \mathcal{O}^{n} \subset \partial \mathcal{O} \cap Z^{n}
$$

Consider a smooth function $f \in C^{\infty}(\mathcal{O})$ and $m>0$.

Definition 3. The function $f$ is called m-admissible, if

i) $0 \leqq f \leqq m$ everywhere, $f \equiv 0$ in a nonempty subdomain of $\mathcal{O}$ and $\left.f\right|_{\partial \mathcal{C}} \equiv m$;

ii) the set $\{z \mid f(z)<m\}$ is bounded. The distance from this set to $\partial \cup$ is $d(f)>0$.

Denote

$$
\operatorname{Supp} f=\{z \mid 0<f(z)<m\} \text {. }
$$

For each $m$-admissible function $f$ we have

$$
\begin{gathered}
\operatorname{dist}\left(f^{-1}(0), \partial \mathcal{O}\right) \geqq d=d(f), \\
\operatorname{dist}(\operatorname{Supp} f, \partial \mathcal{O}) \geqq d .
\end{gathered}
$$

Denote $f_{n}=\left.f\right|_{\mathcal{C}^{n}}$ and consider the corresponding hamiltonian vectorfield $V_{f_{n}}$ in $\mathcal{O}^{n}$. We call a $T$-periodic trajectory of $V_{f_{n}}$ fast if it is not a stationary point and $T \leqq 1$.

Lemma 4. Each fast periodic trajectory is contained in $\operatorname{Supp} f \cap Z^{n}$.

Proof. At each point $z \in \mathcal{O}^{n} \backslash \operatorname{Supp} f$ the function $f_{n}$ takes either its minimal or maximal value. So $V_{f_{n}}(z)=0, z$ is a stationary point, and a fast trajectory cannot pass through it.

We call an admissible function $f$ fast if there exists $n_{0}=n_{0}(f)$ such that for all $n \geqq n_{0}$ the vectorfield $V_{f_{n}}$ has a fast solution.

Now we are in a position to define a capacity $c$ :

Definition 4. For an open nonempty domain $\mathcal{O} \subset Z$ its capacity $c(\mathcal{O})$ equals to

$$
c(\mathcal{O})=\inf \left\{m_{*} \mid \text { each } m \text {-admissible function with } m>m_{*} \text { is fast }\right\} \text {. }
$$

The capacity $c(\mathcal{O})$ depends on the stratification $Z^{1} \subset Z^{2} \subset \cdots$ of the space $Z$, formed by invariant subspaces of the linear operator $J A$. The function $h$ in (2.3) can 
contain a quadratic in $z$ part. Thus the linear operator $A$ is fixed modulo compact linear operators only. Equations (2.4) with fixed "unbounded part" of the operator $A$ forms a natural class of equations which can be studied with the same capacity $c$.

Compact perturbations of the operator $A$ imply perturbations of the basis $\left\{\varphi_{j}^{ \pm}\right\}$. The corresponding stability result for the capacity $c$ can be stated as follows:

Proposition 1. The capacity $c(\mathcal{O})$ of a domain $\mathcal{O}$ will not change if the basis $\left\{\varphi_{j}^{ \pm}\right\}$is replaced by another Hilbert basis Darboux $\left\{\psi_{j}^{ \pm}\right\}$, which is quadratically close to the initial one,

$$
\sum\left\|\varphi_{j}^{ \pm}-\psi_{j}^{ \pm}\right\|^{2}<\infty
$$

For a sketch of a proof see Part 5.

We continue with some trivial properties of the capacity $c$ which can be obtained immediately.

Proposition 2. For nonempty domains $\mathrm{O}_{1}, \mathrm{O}_{2}, \mathcal{O} \subset Z$ and $\tau \neq 0$ we have

a) $c\left(\mathcal{O}_{1}\right) \leqq c\left(\mathcal{O}_{2}\right)$ if $\mathscr{U}_{1} \subset \mathcal{O}_{2}$

b) $c\left(\tau(\mathcal{O})=\tau^{2} c(\mathcal{O})\right.$

Proof. For $m<c\left(\mathcal{O}_{1}\right)$ we can find an $m$-admissible function $f$ in $\mathcal{O}_{1}$ which is not fast. That is there exists an infinite sequence $\left\{n_{j}\right\}$ such that each vectorfield $V_{f_{n_{j}}}$ has no fast periodic trajectory. Let $\tilde{f}$ be the function $f$, extended as $m$ to $\mathcal{O}_{2} \backslash \mathcal{O}_{1}$. The function $\tilde{f}$ is $m$-admissible in $\mathcal{O}_{2}$ and for $n=n_{1}, n_{2}, \ldots$ the vectorfield $V_{\tilde{f}_{n}}$ has no fast solutions, because by Lemma 4 each fast solutions of $V_{\tilde{f}_{n}}$ lies in $\operatorname{supp} \tilde{f} \cap Z^{n}=\operatorname{Supp} f \cap Z^{n}$, so it is a fast solution of $V_{f_{n}}$. Thus $c\left(\mathrm{O}_{2}\right) \geqq m$ and the first assertion follows.

To prove the second we observe that a curve $z(t) \in \mathcal{O}^{n}$ is a $T$-periodic trajectory of $V_{f_{n}}$ if and only if the curve $\tau z(t) \in \tau \mathcal{O}^{n}$ is a $T$-periodic solution of $V_{f_{n}^{r}}$, where $f^{\tau}(y)=\tau^{2} f\left(\tau^{-1} y\right)$ for $y \in \tau \mathcal{O}$. Moreover, the function $f$ is $m$-admissible for $\mathcal{O}$ if and only if $f^{\tau}$ is $\tau^{2} m$-admissible for $\tau \mathcal{O}$.

Proposition 3. For any $\mathcal{O} \subset Z$,

$$
c(\mathcal{O}) \leqq \longdiv { \operatorname { l i m } } c _ { 2 n } ( \mathcal { O } _ { n } ) .
$$

Proof. For $m<c(\mathcal{O})$ there exists an $m$-admissible function $f$ which is not fast. That is, one can find a sequence $\left\{n_{j}\right\}$ such that the vectorfield $V_{f_{n_{1}}}$ has no fast solution. For large $n_{j}$ the function $f_{n_{j}}$ is $m$-admissible in $\mathcal{O}_{n_{j}}$. Thus, $c_{2 n_{j}}\left(\mathcal{O}_{n_{j}}\right) \geqq m$ and the statement follows.

Proposition 4. For any domain $\mathcal{O} \subset Z$ and $\xi \in Z$ we have

$$
c(\mathcal{O})=c(\mathcal{O}+\xi) \text {. }
$$

Proof. Let us denote $\mathcal{O}_{\xi}=\mathcal{O}+\xi$. It is sufficient to check that $c(\mathcal{O}) \leqq c\left(\mathcal{O}_{\xi}\right)$, because $\mathcal{O}=\mathscr{O}_{\xi}-\xi$ so then also $c\left(\mathcal{O}_{\xi}\right) \leqq \mathcal{C}(\mathcal{O})$. We decompose $\xi$ as $\xi=\xi^{n_{0}}+\xi_{n_{0}}$ (see (2.1); $n_{0}$ will be fixed later) and denote $\mathcal{O}_{1}=\mathcal{O}+\xi^{n_{0}}$. Clearly, $c\left(\mathcal{O}_{1}\right)=c(\mathcal{O})$ (see also Lemma 5 below) and $\mathscr{O}_{\xi}=\mathcal{O}_{1}+\xi_{n_{0}}$.

We take any $m$-admissible function $f$ in $\mathcal{O}_{\xi}$ with $m>c(\mathcal{O})$ and wish to check that this function is fast. As $\left\|\xi_{n}\right\| \rightarrow 0$ as $n \rightarrow \infty$, then

$$
\operatorname{dist}\left(\partial \mathcal{O}_{1}, \partial \mathcal{O}_{\xi}\right) \leqq\left\|\xi_{n}\right\| \rightarrow 0 \quad \text { as } n \rightarrow \infty .
$$


So we can find $n_{0}$ so large that $\operatorname{dist}\left(\partial \mathcal{O}_{1}, \partial \mathcal{O}_{\xi}\right) \leqq\left\|\xi_{n_{0}}\right\|<\frac{1}{2} d(f)$. We extend $f$ as $m$ outside $\mathcal{O}_{\xi}$ and denote by $f^{1}$ restriction of the extended function to $\mathcal{O}_{1}$. The new function equals $m$ in the $\frac{1}{2} d(f)$-neighborhood of $\partial \mathcal{O}_{1}$ and vanishes at the set $f^{-1}(0)$ which is contained in $\mathcal{O}_{1} \cap \mathcal{O}_{\xi}$ by (2.2). So it is $m$-admissible and thus $V_{f_{n}^{1}}$ has a fast trajectory in $\mathcal{O}_{1}^{n}$ if $n \geqq n_{0}$ is sufficiently large, because $c\left(\mathcal{O}_{1}\right)<m$. By Lemma 4 this trajectory lies in $\operatorname{Supp} f^{1}=\operatorname{Supp} f \subset \mathcal{O}_{1} \cap \mathcal{O}$. So it also is a fast trajectory of $V_{f_{n}}$. Thus, the function $f$ is fast.

Now we shall show that the capacities of ellipsoids and polydiscs are given by formulas similar to the finite-dimensional formulas (1.3), and that the capacities remain unchanged under some "reasonably wide" class of symplectomorphisms.

Let $\boldsymbol{r}=\left\{r_{1}, r_{2}, \ldots\right\}$ be a sequence such that $0<r_{j} \leqq \infty$ for all $j$ and

$$
0<r:=\inf _{j} r_{j}<\infty .
$$

We define the polydisc $D(\boldsymbol{r})$ and the ellipsoid $B(\boldsymbol{r})$ as follows:

$$
\begin{aligned}
& D(\boldsymbol{r})=\left\{z=\sum p_{j} \varphi_{j}^{+}+q_{j} \varphi_{j}^{-} \mid p_{j}^{2}+q_{j}^{2}<r_{j}^{2} \forall j\right\}, \\
& B(\boldsymbol{r})=\left\{z \mid \sum r_{j}^{-2}\left(p_{j}^{2}+q_{j}^{2}\right)<1\right\} .
\end{aligned}
$$

Clearly,

$$
B(\boldsymbol{r}) \subset D(\boldsymbol{r}) .
$$

Theorem 1. For each sequence $\boldsymbol{r}$ as in (3.4),

$$
c(B(\boldsymbol{r}))=c(D(\boldsymbol{r}))=\pi r^{2} .
$$

Proof of the theorem is given in Part 4 below.

Corollary. For $r>0$

$$
c\left(B_{r}\right)=\pi r^{2} .
$$

From this statement we get nontriviality of the capacity $c$ :

Theorem 2. For each nonempty bounded open domain $\mathcal{O}$ we have

$$
0<c(0)<\infty \text {. }
$$

Proof. We can find $\rho, R>0$ and $\xi \in Z$ such that

$$
B_{\rho}+\xi \subset \mathcal{O} \subset B_{R} \text {. }
$$

Thus by Proposition 4 and the corollary we have $\pi \rho^{2} \leqq c(\mathcal{O}) \leqq \pi R^{2}$.

Now we turn to invariance of the capacity. We start with a trivial observation.

Lemma 5. If a map $F: Z \rightarrow Z$ has the form

$$
F\left(z^{n}, z_{n}\right)=\left(F^{n}\left(z^{n}\right), z_{n}\right), \quad\left(z^{n}, z_{n}\right) \in Z^{n} \times Z_{n},
$$

where $F^{n}$ is a smooth symplectomorphism of $Z^{n}$, then $c(F(\mathcal{O}))=c(\mathcal{O})$ for each domain 0 .

Proof. The map $F$ and its inverse are Lipschitz uniformly in bounded subsets. So $F^{*}$ sends $m$-admissible functions in $F(\mathcal{O})$ to similar ones in $\mathcal{O}$. For $N \geqq n$ it transforms the vectorfield $V_{\tilde{f}_{N}}, \tilde{f}=f \circ F$, to $V_{f_{N}}$. So the classes of admissible and fast functions are preserved by $F$, and the result follows. 
A much more essential property of the capacity $c$ is its invariance with respect to elementary symplectomorphisms.

Theorem 3. For any elementary symplectomorphism $\Phi$ and any domain $\mathcal{O}$ we have

$$
c(\mathcal{O})=c(\Phi(\mathcal{O})),
$$

provided that the map $\Phi^{-1}: \Phi(\mathcal{O}) \rightarrow \mathcal{O}$ is bounded (it sends bounded sets to bounded sets).

Proof of the theorem is given in Part 5.

Remarks. 1) The last assumption of the theorem holds trivially if the set $\mathcal{O}$ is bounded.

2) If the map $\Phi^{-1}$ is not bounded, we can only state that $c(\mathcal{O}) \leqq c(\Phi(\mathcal{O})$ ).

3) We need the compactness assumptions $\nabla h \in C^{1}\left(\mathbb{R} \times Z_{-}, Z_{+}\right)$and $\left(2.8^{\prime \prime}\right)$ only to get the decomposition (2.14) which is essentially equivalent to compactness of the operator $S_{t}$ in (2.9). So what we really need to prove, that the maps $S_{t}$ preserve the capacity, is their smoothness jointly with the decomposition (1) from the introduction.

Corollary (the Squeezing Theorem). If an elementary symplectomorphism $\Phi$ sends a ball

$$
\{z \in Z \mid\|z-\bar{z}\|<r\}
$$

to a cylinder

$$
\left\{z=\sum p_{j} \varphi_{j}^{+}+q_{j} \varphi_{j}^{-} \mid\left(p_{j_{0}}-\bar{p}\right)^{2}+\left(q_{j_{0}}-\bar{q}\right)^{2}<R^{2}\right\}
$$

(the index $j_{0}$ is fixed) then $r \leqq R$.

\section{Proof of Theorem 1}

We shall check that

$$
c(B(\boldsymbol{r})) \geqq \pi r^{2}
$$

and

$$
c(D(r)) \leqq \pi r^{2} .
$$

After this the result follows from (3.5) and Proposition 2.

1) To prove (4.1) we fix any $m<\pi r^{2}$ and produce an $m$-admissible function $F$ in $B(r)$, which is not fast. The construction of the function $F$, given below, directly follows [HZ1, HZ2].

The ball $B=B_{r}(Z)$ is contained in $\mathrm{B}(\boldsymbol{r})$. Fix any $\gamma \in\left(m, \pi r^{2}\right)$. At the ball $B$ the function

$$
u \mapsto \frac{\gamma}{r^{2}}\|u\|^{2}
$$

varies from zero to $\gamma$. We can find $\rho>0, \gamma_{1}<\gamma$ and a smooth function $f(t)$ such that $0 \leqq f^{\prime}(t) \leqq 1,0 \leqq f \leqq m$ and

$$
f(t)= \begin{cases}m & \text { if } t \geqq \gamma_{1} \\ 0 & \text { if } t \leqq \rho .\end{cases}
$$


Define

$$
F(z)=f\left(\frac{\gamma}{r^{2}}\|u\|^{2}\right) .
$$

The function $F$ is smooth in $Z$, it vanishes near zero and equals $m$ outside the ball $B$ and in the set $\left\{u \mid\|u\|>r \sqrt{\gamma_{1} / \gamma}\right\}$. Thus, $F \equiv m$ in some $\varepsilon$-neighborhood of $\times \partial B_{r}$ in $Z$, and so it is $m$-admissible.

We wish to show that the function $F$ is not fast. We introduce in $Z^{n}$ the action-angle variables $I, \varphi$,

$$
I_{j}=\frac{1}{2}\left(p_{j}^{2}+q_{j}^{2}\right), \quad \varphi_{j}=\arctan \left(q_{j} / p_{j}\right)
$$

where $\left\{\left(p_{j}, q_{j}\right)\right\}$ are coordinates with respect to the basis $\left\{\varphi_{j}^{ \pm} \mid 1 \leqq j \leqq n\right\}$ in $Z^{n}$. Then

$$
F_{n}=f\left(\sum \frac{2 \gamma}{r^{2}} I_{j}\right)
$$

So

$$
\dot{\varphi}_{j}=f^{\prime} \frac{2 \gamma}{r^{2}} \leqq \frac{2 \gamma}{r^{2}}<2 \pi
$$

and $T>1$ for each nontrivial $T$-periodic solution of $V_{F_{n}}$ in $Z^{n}$.

Thus, $c(B(\boldsymbol{r})) \geqq m$ and (4.1) is proven.

2) Denote $D(\boldsymbol{r})=0$. To prove (4.2) we fix some $m>\pi r^{2}$ and take any $m$ admissible function $f$ in $\mathcal{O}$. As the interior of the set $f^{-1}(\mathcal{O})$ is not empty, then the same is true for the set $f^{-1}(0) \cap Z^{n}$ with sufficiently large $n$. As $\partial \mathcal{O}^{n} \subset \partial \mathcal{O}$, then the smooth function $f_{n}=\left.f\right|_{\mathcal{C}^{n}}$ equals $m$ near $\partial \mathcal{O}^{n}$. So it is $m$-admissible.

We know (see (1.4)) that $c_{n}\left(\mathcal{O}_{n}\right)=\pi \min _{1 \leqq j \leqq n} r_{j}^{2}$. So $c_{n}\left(\mathcal{O}_{n}\right) \rightarrow \pi r^{2}$ and $c_{n}\left(\mathcal{O}_{n}\right)<m$ for large $n$. For such $n$ 's the vectorfield $V_{f_{n}}$ has a fast periodic trajectory, and the function $f$ is fast.

\section{Proof of Theorem 3 and Proposition 1}

We start with

Lemma 6. If $\Phi: \mathcal{O} \rightarrow Q \subset Z$ is an elementary symplectomorphism such that the inverse map $\Phi^{-1}$ is bounded then the symplectomorphism $\Phi^{-1}: Q \rightarrow \mathcal{O}$ also is elementary.

Proof. If $\Phi$ is the $S_{T^{-}}$flow for some Eq. (2.4) satisfying (2.2), (2.8)-(2.8"), then $\Phi^{-1}$ is the $\tilde{S}_{T}$-flow for the equation with the hamiltonian $\tilde{f}$,

$$
\tilde{f}=-\frac{1}{2}\langle A z, z\rangle-h(z, T-t),
$$

which also meets (2.2), (2.8)-(2.8").

The flow $\widetilde{S_{t}}(z)$ is well-defined for $z \in Q, 0 \leqq t \leqq T$; it is bounded by the lemma's assumption. The flow-map $S_{T}$ is well-defined for $u(0)$ from some neighborhood of $\overline{\mathcal{O}}$ (see Definitions 1,2 ). Its image contains a neighborhood of $\bar{Q}$, where the flow $\tilde{S}_{t}$ is 
well-defined for $0 \leqq t \leqq T$. Thus, the hamiltonian $f$ is admissible for $0 \leqq t \leqq T$, $u_{0} \in Q$, and the assertion follows.

Proof of Theorem 3. We denote $Q=\Phi(\mathcal{O})$ and take any $m$-admissible function $f$ in $Q$, where $m>c(\mathcal{O})$. If we can prove that the function $f$ is fast, then $c(Q) \leqq c(\mathcal{O})$ and the result follows, because the suplectomorphism $\Phi^{-1}$ also is elementary by Lemma 6 and so $c(\mathcal{O}) \leqq c(Q)$ as well.

The set $\operatorname{Supp} f$ is contained in some ball $B_{R}$. We take $R_{1}=R+d(f)$ and denote $Q^{\prime}=Q \cap B\left(R_{1}\right), \mathcal{O}^{\prime}=\Phi^{-1}\left(Q^{\prime}\right)$. By our assumptions the set $\mathcal{O}^{\prime}$ is bounded, $\mathcal{C}^{\prime} \subset B\left(R^{\prime}\right)$, and by Proposition 2 ,

$$
c\left(\mathcal{O}^{\prime}\right) \leqq c(\mathcal{O}) .
$$

We apply Lemma 3 with $N$ so large that $\varepsilon<\frac{1}{2} d(f)$ and denote by $\mathcal{O}_{1}, \mathcal{O}_{2}$ the intermediate domains which arise from the decomposition (2.13):

$$
\mathcal{O}^{\prime} \stackrel{I+\widetilde{\Phi}_{N}}{\longrightarrow} \mathcal{O}_{1} \stackrel{I+\tilde{\Phi}_{t}}{\longrightarrow} \mathcal{O}_{2} \stackrel{e^{\Gamma J A}}{\longrightarrow} Q^{\prime}
$$

We also denote

$$
f_{2}=\left.f \circ e^{T J A}\right|_{c_{2}} .
$$

As the map $e^{T J A}$ is an isometry, then

$$
\operatorname{dist}\left(\operatorname{Supp} f_{2}, \partial \mathscr{O}_{2}\right)=d=d(f)
$$

and the function $f_{2}$ is $m$-admissible in $\mathcal{O}_{2}$.

We extend $f_{2}$ as $m$ outside $\mathcal{O}_{2}$ and get a smooth function $f_{2}^{\text {ext }}$ in $Z$. Denote by $f$ its restriction to $\mathscr{O}_{1}$. The $\varepsilon$-neighborhood of $\partial \mathcal{O}_{1}$ is contained in the $2 \varepsilon$-neighborhood of $\partial \mathcal{O}_{2}$, where $f_{2}^{\text {ext }} \equiv m$ as $\varepsilon<\frac{1}{2} d$. By (3.2) $\tilde{f}^{-1}(0)=f_{2}^{-1}(0) \subset \mathcal{O}_{1} \cap \mathcal{O}_{2}$. So the function $\tilde{f}$ is $m$-admissible in $\mathcal{O}_{1}$.

By Lemma $5 c\left(\mathcal{O}_{1}\right)=c\left(\mathcal{O}^{\prime}\right)$. Thus

$$
c\left(\mathcal{O}_{1}\right)=c\left(\mathcal{O}^{\prime}\right) \leqq c(\mathcal{O})<m,
$$

and the function $\tilde{f}$ is fast. So for each $n \gg 1$ the vectorfield $V_{\tilde{f}_{n}}$ has in $\mathcal{O}_{1}^{n}$ a fast solution. By Lemma 4 this solution lies in Supp $\tilde{f}$, which equals $\operatorname{Supp} f_{2}$ by (3.3). Therefore the solution is also a fast trajectory of $V_{f_{2} n}$, the function $f_{2}$ is fast as well as the function $f$ and the result follows.

Now we go to a proof of Proposition 1. The linear transformation $L$ which corresponds coefficients of a vector's decomposition in the basis $\left\{\varphi_{j}^{ \pm}\right\}$to its coefficients in the basis $\left\{\psi_{j}^{ \pm}\right\}$is a symplectomorphism, compact-close to identity. Thus we can decompose it as

$$
L=L_{2} \circ L_{1},
$$

where $L_{1}$ changes first $2 N$ coordinates only and $L_{2}$ is close to identity:

$$
\left\|L_{2}-I\right\|_{Z, Z} \leqq \varepsilon=\varepsilon(N),
$$

where $\varepsilon(N) \rightarrow 0$ as $N \rightarrow \infty$. For the map $L$ we have got an analog of the decomposition (2.14). So we can complete the proof by repeating the arguments we used above to prove Theorem 3 . 


\section{Examples}

\subsection{Schrödinger Equation}

Take $Z=L_{2}\left(\mathbb{T}^{n} ; \mathbb{C}\right), n \geqq 1, \mathbb{T}^{n}=\mathbb{R}^{n} / 2 \pi \mathbb{Z}^{n}$, with the scalar product

$$
\langle u, v\rangle=\operatorname{Re} \int_{\mathbb{T}^{n}} u \bar{v} d x /(2 \pi)^{n}
$$

and the symplectic form

$$
\omega[u, v]=-\operatorname{Im} \int u \bar{v} d x /(2 \pi)^{n}
$$

(corresponding to the operator $J u=i u$ ).

Consider the operator

$$
A=-\Delta+V(x)
$$

with a smooth periodic potential $V(x)$ and denote by $\left\{\varphi_{j}\right\}$ the complete system of its real eigenfunctions,

$$
A \varphi_{j}(x)=\lambda_{j} \varphi_{j}(x) \forall j ;\left\langle\varphi_{j}, \varphi_{k}\right\rangle=\delta_{j k} \forall j, k .
$$

For the basis $\left\{\varphi_{j}^{ \pm}\right\}$of $Z$ we take

$$
\varphi_{j}^{+}=\varphi_{j}(x), \quad \varphi_{j}^{-}=i \varphi_{j}(x) .
$$

Consider the hamiltonian

$$
f=\frac{1}{2}\langle A u, u\rangle+\frac{1}{2} \int G(U, \bar{U} ; x, t) d x /(2 \pi)^{n}, \quad U=u * \xi,
$$

where $G$ is a real-valued smooth function and $u * \xi$ is the convolution with a fixed real-valued function $\xi \in H^{1}\left(\mathbb{T}^{n}\right)$. Denote the second term in (6.1) as $h_{t}(u)$. Then

$$
\begin{aligned}
h_{t}(u+\delta v) & =h_{t}(u)+\frac{\delta}{2} \int((\partial / \partial U) G V+(\partial / \partial \bar{U}) G \bar{V}) d x+O\left(\delta^{2}\right) \\
& =h_{t}(u)+\delta\langle(\partial / \partial \bar{U} G) * \xi, v\rangle+O\left(\delta^{2}\right), V=v * \xi .
\end{aligned}
$$

So

$$
\nabla h_{t}(u)=\xi * \partial / \partial \bar{U} G(U, \bar{U} ; t, x),
$$

and the equation with the hamiltonian $f$ has the form

$$
-i \dot{u}=-\Delta u+V(x) u+(\partial / \partial \bar{U} G(U, \bar{U} ; t, x)) * \xi, \quad U=u * \xi .
$$

Take $Z^{-}=H^{-1}\left(\mathbb{T}^{n} ; \mathbb{C}\right), Z^{+}=H^{1}\left(\mathbb{T}^{n} ; \mathbb{C}\right)$, where the Sobelev space $Z^{+}$is given the norm $\|u\|_{1}^{2}=\langle(c \mathbf{1}+A) u, u\rangle$ with sufficiently large $c$. Then

$$
Z^{+}<Z<Z^{-} \text {. }
$$

The map

$$
Z^{-} \rightarrow Z^{+}, \quad u \mapsto \xi *(\partial / \partial \bar{U} G(U, \bar{U} ; t, x)),
$$

is smooth. So the relations $(2.8)-\left(2.8^{\prime \prime}\right)$ hold, the hamiltonian equation (6.2) is admissible and its flow-maps $S_{t}$ preserve the capacities $c(\mathcal{O})$ of open domains $\mathcal{O} \subset Z$ till the blow-up time. The blow-up will never occur, in particular, if the function $\partial / \partial \bar{U} G(U, \bar{U} ; t, x)$ is bounded or if $G(U, \bar{U} ; t, x)=f\left(|U|^{2} ; t, x\right)$ for some real function $f$. 
If for some $w \in Z$ we denote

$$
\boldsymbol{B}_{\rho}=\{u(x) \mid\|u-w\|<\rho\},
$$

then $c\left(\boldsymbol{B}_{\rho}\right)=\pi \rho^{2}$ by Theorem 1 and Proposition 4 . If $j_{0} \in \mathbb{N}$, if $\bar{p}, \bar{q}$ are some reals and

$\boldsymbol{\Pi}_{R}=\left\{u=\sum p_{j}^{+} \varphi_{j}^{+}+p_{j}^{-} \varphi_{j}^{-} \equiv \sum\left(p_{j}^{+}+i p_{j}^{-}\right) \varphi_{j}(x)\left|p_{j_{0}}-\bar{p}\right|^{2}+\left|q_{j_{0}}-\bar{q}\right|^{2}<R^{2}\right\}$, then $c\left(\boldsymbol{\Pi}_{R}\right)=\pi R^{2}$. So in particular

$$
S_{t}\left(\boldsymbol{B}_{\rho}\right) \subset \boldsymbol{\Pi}_{\boldsymbol{R}} \quad \text { implies } \quad R \geqq \rho .
$$

That means that if the initial wave-function $u(0, x)$ was known with the accuracy $\rho$ and the system evolves in accordance with (6.2), then each Fourier coefficient $\left(p_{j_{0}}^{+}+i p_{j_{0}}^{-}\right)(t)$ never can be measured with an accuracy better than $\rho^{6}{ }^{6}$

\subsection{Nonlinear String Equation}

Consider a nonlinear string equation

$$
\ddot{u}=u_{x x}-u-f(u ; t, x), \quad u=u(t, x),^{7}
$$

where $x \in \mathbb{T}^{1}=\mathbb{R} / 2 \pi \mathbb{Z}$ and $f$ is a smooth function which has at most a polynomial growth in $u$, as well as its $u$ - and $t$-derivatives:

$$
\left|\frac{\partial^{a}}{\partial u^{a}} \frac{\partial^{b}}{\partial t^{b}} f(u ; t, x)\right| \leqq C_{k}(1+|u|)^{M_{k}} \quad \text { for } a+b=k \text { and all } k \geqq 0,
$$

where positive constants $C_{k}$ are bounded for bounded $t$ and nonnegative $M_{k}$ 's are $t$-independent. We denote by $B$ the operator $B=\left(-\partial^{2} / \partial x^{2}+1\right)^{1 / 2}$ and write the equation in the form

$$
\begin{aligned}
& \dot{u}=-B v, \\
& \dot{v}=B u+B^{-1} f(u ; t, x) .
\end{aligned}
$$

Define the phase-space $Z$ of Eq. (6.5) as $Z=H^{1 / 2} \times H^{1 / 2}$, where $H^{1 / 2}=H^{1 / 2}\left(\mathbb{T}^{1}\right)$ is the Sobolev space with the scalar product

$$
\left\langle u_{1}, u_{2}\right\rangle=\int_{0}^{2 \pi} B u_{1}(x) u_{2}(x) d x / 2 \pi
$$

For the symplectic Hilbert basis $\left\{\varphi_{j}^{ \pm} \mid j \in \mathbb{Z}\right\}$ of $Z$ we take

$$
\varphi_{j}^{+}=\left(j^{2}+1\right)^{-1 / 4}\left(\varphi_{j}(x), 0\right), \quad \varphi_{j}^{-}=\left(j^{2}+1\right)^{-1 / 4}\left(0, \varphi_{j}(x)\right),
$$

where

$$
\varphi_{j}(x)= \begin{cases}\sqrt{2} \sin j x, & j>0 \\ \sqrt{2} \cos j x, & j \leqq 0\end{cases}
$$

\footnotetext{
${ }^{6}$ We remark that this statement is trivial if $G=f\left(|U|^{2} ; t, x\right)$ and $w=0, \bar{p}=\bar{q}=0$ in the definitions of $\boldsymbol{B}_{\rho}$ and $\boldsymbol{\Pi}_{R}$ because in such a case the flow-maps preserve the norm in $Z$.

${ }^{7}$ We write the nonlinear term in the form $u-f(u ; t, x)$ for convenience; possibly $\partial / \partial u f(0 ; t, x) \neq 0$.
} 
We define the operator $J$ as in Part 2 (i.e., $J \varphi_{j}^{ \pm}=\mp \varphi_{j}^{\mp}$ ). Then

$$
J(u, v)=(-v, u) .
$$

Consider the functional $h_{t}$ in $Z$,

$$
h_{t}(u, v)=\int_{0}^{2 \pi} F(u(x) ; t, x) d x / 2 \pi, \quad F=\int_{0}^{u} f d u .
$$

Its gradient $\nabla h_{t}$ in the space $Z$ equals

$$
\nabla h_{t}=\left(B^{-1} f(u,(x) ; t, x), 0\right) .
$$

So if we choose $A=B \times B$, and denote $U=(u, v)$, then Eq. (2.4),

$$
\dot{U}=J\left(A U+\nabla h_{t}(U)\right),
$$

coincides with (6.5).

For the space $Z_{-}$we take $Z_{-}=H^{1 / 2-\theta} \times H^{1 / 2-\theta}$ with some $0<\theta<\frac{1}{2}$. Clearly $Z_{+}=H^{1 / 2+\theta} \times H^{1 / 2+\theta}$ and

$$
Z_{+}<Z<Z_{-} \text {. }
$$

Lemma. The functional $h$ meets the compactness assumptions (2.8)-(2.8"), if $\theta>0$ is sufficiently small.

Proof. To check the first relation in (2.8) we prove that the map

$$
H^{1 / 2-\theta} \times \mathbb{R} \rightarrow L_{2}, \quad(u(\cdot), t) \mapsto f(u(\cdot), t ; \cdot),
$$

is $C^{1}$-smooth. This statement follows from (6.4) and the Sobolev embedding theorem. In particular,

$$
\begin{aligned}
\mid f(u(x)+v(x)) & -\left.f(u(x))\right|_{L_{2}} ^{2} \leqq C_{1}^{2}\left|(1+|u|)^{M_{1}} v\right|_{L_{2}}^{2} \\
& \leqq\left. C_{1}^{2}\left|(1+|u(x)|)_{L_{2 M_{1}+2}}^{2 M_{1}}\right| v\right|_{L_{2 M_{1}+2}} ^{2} \\
& \leqq C^{\prime}\left(1+\|u\|_{1 / 2-\theta}\right)^{2 M_{1}}\|v\|_{1 / 2-\theta}^{2}
\end{aligned}
$$

so the map (6.7) is continuous, etc.

The second relation in (2.8) follows from smoothness of the map

$$
H^{1 / 2} \times \mathbb{R} \rightarrow L_{2}, \quad(u(x), t) \mapsto f(u(\cdot) ; t, \cdot),
$$

which in its turn results from (6.4) because the space $H^{1 / 2}$ is embedded in each space $L_{p}, p<\infty$.

The relations $\left(2.8^{\prime}\right),\left(2.8^{\prime \prime}\right)$ both follow from the continuity of the map (6.7).

Thus Eq. (6.5) in the form (2.4) is admissible in the space $Z$ and its flow-maps $S_{t}$ preserve the capacity $c(\mathcal{O})$ of a domain $\mathcal{O} \subset Z$ if $\tau(\mathcal{O}) \geqq t$. So the "squeezing" (6) (see Introduction) is impossible for the nonlinear wave equation in the phase space $H^{1 / 2} \times H^{1 / 2}$ till the blow-up time.

We remark that solutions of (6.3) never blow-up in particular, if $M_{0}=0$ (i.e., if the function $f$ is bounded and meets (6.4)). This readily results from representing the equation in the form (6.5). 


\subsection{Nonlinear Wave Equation}

The equation

$$
\ddot{u}=\Delta u-u-f(u ; t, x), \quad x \in \mathbb{T}^{n}, \quad n \geqq 2,
$$

also can be written in the form (6.5) with $Z=H^{1 / 2}\left(T^{n}\right) \times H^{1 / 2}\left(T^{n}\right)$ and $B=(-\Delta+1)^{1 / 2}$. Now it is much more difficult to satisfy $(2.8)-\left(2.8^{\prime \prime}\right)$. The only example we know is the quadratic in $u$ function $f$ for $n=2$. So the only admissible multidimensional equation (6.8) we know is the equation

$$
\ddot{u}=\Delta u+a(t, x) u+b(t, x) u^{2}, \quad x \in \mathbb{T}^{2} .
$$

We also remark that the assumptions (2.8)-(2.8") are fulfilled if the nonlinear term is "smoothed out" and Eq. (6.8) takes the form

$$
\ddot{u}=\Delta u-u+\xi * g^{\prime}(u * \xi, t), \quad x \in \mathbb{T}^{n},
$$

where $\xi \in H^{1}\left(\mathbb{T}^{n}\right)$. Now

$$
h(u, v)=\int_{\mathbb{T}^{n}} g(u * \xi, t) /(2 \pi)^{n},
$$

and one again can rewrite the equation in the form (2.4) with $Z=$ $H^{1 / 2}\left(\mathbb{T}^{n}\right) \times H^{1 / 2}\left(\mathbb{T}^{n}\right)$, choose $Z_{-}=L_{2} \times L_{2}, Z_{+}=H^{1} \times H^{1}$ and see that the equation is admissible.

\subsection{Membrane Equation}

Consider the nonlinear membrane equation

$$
\ddot{u}=-\left(\Delta^{2} u+u+f(u ; t, x)\right), \quad x \in \mathbb{T}^{2},
$$

where the function $f$ is the same as in (6.4). We denote $B=\left(\Delta^{2}+1\right)^{1 / 2}$ and rewrite Eq. (6.9) in the form (6.5). Take $Z=H^{1} \times H^{1}$, where $H^{1}=H^{1}\left(\mathbb{T}^{2}\right)$ is given the norm

$$
\|u\|_{1}^{2}=\int_{\mathbb{T}^{2}} B u(x) u(x) d x /(2 \pi)^{2} .
$$

Now Eq. (6.9) (in the form (6.5)) is a hamiltonian equation (2.4) with

$$
h=\int_{\mathbb{T}^{2}} F(u(x) ; t, x) d x /(2 \pi)^{2}, \quad F=\int_{0}^{u} f d u .
$$

We take $Z_{-}=H^{1-\theta} \times H^{1-\theta}$ with sufficiently small positive $\theta$. The functional $h$ meets (2.8)-(2.8") and Eq. (6.9) is admissible in the phase-space $Z$. So its flow-maps $S_{t}$ preserve capacities of subdomains in $Z$.

\section{Appendix 1. Local Solvability of Equation (2.4)}

We consider Eq. (2.4) satisfying (2.2), (2.8)-(2.8").

Theorem . For each positive $T$ and $R$ there exists positive $\tau=\tau(T, R)$ such that for $v \in B_{R}$ and $0 \leqq t_{1}<T$ the unique solution $z(t)$ of $(2.4)$ with $z\left(t_{1}\right)=v$ exists for $t_{1} \leqq t \leqq t_{2}=\min \left(T, t_{1}+\tau\right)$. Moreover, $z(t) \in B_{2 R}$ and the operators $S_{t_{1}, t}(v)=z(t)$ define smooth diffeomorphisms $S_{t_{1}, t}: B_{R} \rightarrow Z$. 
Proof. In the Banach space of continuous curves $\left[t_{1}, t_{2}\right] \rightarrow Z$ vanishing at $t_{1}$, consider the ball $\boldsymbol{B}$ of the radius $2 R$ centered at zero, and the map

$$
F: \boldsymbol{B} \rightarrow \boldsymbol{B}, \jmath(t) \mapsto \int_{t_{1}}^{t} e^{(t-\tau) J A} J \nabla h_{\tau}\left(e^{\left(\tau-t_{1}\right) J A} v+z(\tau)\right) d \tau .
$$

By (2.8) the map

$$
[0, T] \times B_{2 R} \rightarrow Z, \quad(t, \precsim) \mapsto \nabla h(z ; t)
$$

is bounded and Lipschitz; the map $e^{(t-\tau) J A} J$ is a linear isometry of $Z$. So $F$ defines a contraction of the ball $\boldsymbol{B}$ if $\left|t_{2}-t_{1}\right| \leqq \tau$, where $\tau$ is sufficiently small. Therefore the map $F$ has the only stationary point $z(\cdot)$ which defines the unique solution $z(t)$ of (2.6),

$$
z(t)=e^{\left(t-t_{1}\right) J A} v+z(t) .
$$

We skip a traditional proof of the fact that the maps $S_{t_{1}, t}: B_{R} \rightarrow B_{2 R}$ are smooth. ${ }^{8}$ They are diffeomorphisms because the maps $S_{t, t_{1}}$ are their inverse.

\section{Appendix 2. Proof of Lemma 3}

Proof. We can modify the hamiltonian $h$ as in (2.13). After this modification the map $\Phi$ is $S_{T}$-flow for some Eq. (2.4), where

$$
h_{t}(u) \equiv 0 \quad \text { for } \quad\|u\|_{-}>\hat{R}
$$

with some $\hat{R}>R^{\prime}$.

We shall use the following statement which will be proven later.

Lemma. For $|t| \leqq T$, any $\tilde{R}$ and $u, v \in B_{\tilde{R}}(Z)$ we have

$$
\left\|\nabla h_{t}(u)-\nabla h_{t}(v)\right\| \leqq C(\tilde{R})\|u-v\|,
$$

and

$$
\left\|\nabla h_{t}(u)-\Pi^{N} \nabla h_{t}(u)\right\| \leqq \varepsilon_{1}(N),
$$

where $\varepsilon_{1}(N) \rightarrow 0$ as $N \rightarrow \infty$.

Now we set $h_{N}=h_{t} \circ \Pi^{N}$. Then

$$
\nabla h_{N}=\Pi^{N} \circ \nabla h_{t} \circ \Pi^{N} .
$$

We define $\Phi_{N}$ as the time $T$ flow $S_{T}^{N}$ of the equation

$$
\dot{v}=J\left(A v+\nabla h_{N}(v)\right),
$$

or, equivalently, $v=\left(v^{N}, v_{N}\right)$ and

$$
\dot{v}^{N}=J\left(A v^{N}+\Pi^{N} \nabla h_{t}\left(v^{N}\right)\right), \quad \dot{v}_{N}=J A v_{N} .
$$

As $\nabla h\left(v^{N}\right)=0$ for $\left\|v^{N}\right\|_{-}>\hat{R}$ and $\left\|v_{N}(t)\right\|_{-}=$const, then solutions of (A4) cannot leave the ball $B_{2 \hat{R}}\left(Z_{-}\right)$starting from $B_{R}\left(Z_{-}\right)$. Therefore by $\left(2.8^{\prime \prime}\right)$,

$$
\left\|\nabla h_{N}(v(t))\right\| \leqq C(\hat{R})
$$

${ }^{8}$ Warning: the map $\left[t_{1}, t_{2}\right] \times B_{R} \rightarrow B_{2 R},(t, u) \mapsto S_{t_{1}, t}(u)$ is not smooth in $t$. 
and for $0 \leqq t \leqq T$ solutions $v(t)$ with $v(0)$ in $B_{R}(Z)$ do not leave some ball $B_{\tilde{R}}(Z)$. So the flow $S_{t}^{N}$ is well-defined for $0 \leqq t \leqq T$ and sends bounded sets to bounded sets.

By Lemma 1

$$
S_{T}^{N}=\Phi_{N}=e^{T J A}\left(I+\tilde{\Phi}_{N}\right),
$$

where $\left(I+\widetilde{\Phi}_{N}\right)$ is a smooth symplectomorphism (as well as $S_{T}^{N}$ and $\left.e^{T J A}\right)$ and $\widetilde{\Phi}_{N}$ has the form (2.16). Besides

$$
\left(I+\widetilde{\Phi}_{N}\right)^{-1}=S_{T, 0}^{N} \circ e^{T J A}
$$

is a smooth bounded map of $Z$.

Next we estimate the difference $\Phi-\Phi_{N}$. We denote $w(t)=u(t)-v(t)$, where $u(t)$ and $v(t)=\left(v^{N}, v_{N}\right)(t)$ are solutions of (2.4) and (A4) with $u(0)=v(0)=u$.

For $w(t)$ we get the equation

$$
\dot{w}-J A w=J\left(\nabla h_{t}(u)-\nabla h_{t}(v)+\nabla h_{t}(v)-\nabla h_{N}(v)\right) \equiv \Delta(t), \quad w(0)=0 .
$$

By (A2), (A3) $\|\Delta(t)\| \leqq C\|u(t)-v(t)\|+\varepsilon_{1}(N)$. So

$$
\|w(t)\| \leqq \int_{0}^{t}\|\Delta(\tau)\| d \tau \leqq C \int_{0}^{t}\|w(\tau)\| d \tau+t \varepsilon_{1}(N) .
$$

Now Gronwall's lemma implies that

$$
\|w(t)\| \leqq C_{1} \varepsilon_{1}(N) e^{C t} .
$$

As

$$
\Phi-\Phi_{N}=e^{T J A}\left(\tilde{\Phi}-\tilde{\Phi}_{N}\right)
$$

and $\left(\Phi-\Phi_{N}\right) u=w(T)$, then by the last estimate

$$
\left\|\left(\tilde{\Phi}-\tilde{\Phi}_{N}\right)(u)\right\| \leqq C \varepsilon_{1}(N) \underset{N \rightarrow \infty}{\longrightarrow} 0
$$

for $u$ in $B_{R}(Z)$.

We can write $\Phi$ as

$$
\begin{aligned}
\Phi & =e^{T J A}\left(I+\tilde{\Phi}_{N}+\left(\tilde{\Phi}-\tilde{\Phi}_{N}\right)\right)\left(I+\tilde{\Phi}_{N}\right)^{-1}\left(I+\tilde{\Phi}_{N}\right) \\
& =e^{T J A}\left(I+\left(\tilde{\Phi}-\tilde{\Phi}_{N}\right) \circ\left(I+\tilde{\Phi}_{N}\right)^{-1}\right)\left(I+\tilde{\Phi}_{N}\right) .
\end{aligned}
$$

So we get the representation (2.14) with $\tilde{\Phi}_{\varepsilon}=\left(\widetilde{\Phi}-\widetilde{\Phi}_{N}\right)\left(I+\widetilde{\Phi}_{N}\right)^{-1}$. The estimate (2.15) with large enough $N$ results from (A6) and (2.16) follows from the very definition of the hamiltonian $h_{N}$.

Proof of Lemma. The ball $B_{\tilde{R}}(Z)$ is precompact in $Z_{-}$. So by (2.8),

$$
\left\|\nabla h_{t}(u)-\nabla h_{t}(v)\right\| \leqq \sup _{\xi \in B_{\tilde{R}}(Z)|t| \leqq T} \sup _{|t|}\left\|d \nabla h_{t}(\xi)\right\|_{Z_{-}, Z_{+}}\|u-v\| \leqq C(\tilde{R})\|u-v\|,
$$

and (A2) follows.

As the set $K=\bigcup_{|t| \leqq T} \nabla h_{t}\left(B_{\tilde{R}}\right)$ is precompact in $Z_{+}$and in $Z$, then (A3) results from the following statement:

$$
\sup _{u \in K}\left\|u-\Pi^{N} u\right\| \rightarrow 0 \quad \text { as } \quad N \rightarrow \infty .
$$


To prove (A7) we suppose that the convergence does not hold. Then we can find a sequence $\left\{u_{(n)}\right\} \subset K$ such that $\left\|\left(I-\Pi^{n}\right) u_{(n)}\right\| \geqq \varepsilon>0$. As $K$ is precompact, then $u_{\left(n_{j}\right)} \rightarrow u \in Z$. For $n_{j} \gg 1$ we have

$$
\left\|\left(I-\Pi^{n_{j}}\right) u\right\|<\varepsilon / 2, \quad\left\|u_{\left(n_{j}\right)}-u\right\|<\varepsilon / 2 .
$$

So $\|\left(\left(I-\Pi^{n_{j}}\right) u_{\left(n_{j}\right)} \|<\varepsilon\right.$ and we get a contradiction which proves (A7).

Acknowledgements. I am indebted to H. Hofer and E. Zehnder for presenting me with a copy of manuscript of their book [HZ2] and to E. Zehnder for discussions. I am thankful to the FIM (ETH, Zürich), where this paper was done and typed, for its hopspitality, and am indebted to Rahel Boller for her excellent typing.

\section{References}

[A] Arnold, V.I.: First steps in symplectic topology. Uspekhi Mat. Nauk 41:6, 3-18 (1986); English transl. in Russ. Math. Surv. 41:6, 1-21 (1986).

[Bo] Bourgain, J.: Periodic nonlinear Schrödinger equation and invariant measures. To apper in Comm. Math. Phys.

[BK] Bobenko, A.I., Kuksin, S.B.: The nonlinear Klein-Gordon equation on an interval as a pertured Sine-Gordon equation. Submitted to Comment. Math. Helv.

[F] Friedlander L.: An invariant measure for the equation $u_{t t}-u_{x x}+u^{3}=0$. Commun. Math. Phys. 98, 1-16 (1985)

[G] Gromov M.: Pseudo-holomorphic curves on almost complex manifolds. Invent. Math. 82, 307-347 (1985)

[HZ1] Hofer, H., Zehnder, E. : A new capacity for symplectic manifolds. In: Analysis et cetera. P. Rabinowitz, E. Zehnder (eds.) New York: Academic Press, 1990, pp.405-428.

[HZ2] Hofer, H., Zehnder, E.: Symplectic invariants and Hamiltonian dynamics. Birkhäuser, 1994

[K] Kuksin, S.B.: Nearly integrable infinite-dimensional Hamiltonian systems. Lect. Notes Math., 1556, Berlin Heidelberg New York

[P] Pomeau, Y.: Asymptotic time behavior of nonlinear classical field equations. Nonlinearity 5, 707-720 (1992)

[Za] Zakharov, V.E.: Hamiltonian formalism for waves in nonlinear media with a disperse phase. Izv. Vysch. Uchebn. Zaved. Radiofiz. 17, 431-453 1974; English transl. in Radiophysics and Quantum Electronics 17 (1974) 